\title{
Morphology influence on the mechanical stress response in bendable organic field-effect transistors with solution-processed semiconductor
}

Stefano Lai ${ }^{*}{ }^{+}$and Inés Temiño ${ }^{+}$, Tobias Cramer, Freddy G. del Pozo, Beatrice Fraboni, Piero Cosseddu*, Annalisa Bonfiglio and Marta Mas-Torrent*

Dr. S. Lai, Prof. Piero Cosseddu, Prof. A. Bonfiglio

Department of Electrical and Electronic Engineering, University of Cagliari, Piazza d'Armi, 09134, Cagliari, Italy

E-mail: stefano.lai@diee.unica.it, piero.cosseddu@diee.unica.it

I. Temiño, Dr. F. G. del Pozo, Dr. M. Mas-Torrent

Institut de Ciència de Materials de Barcelona (ICMAB-CSIC) and Networking Research

Center on Bioengineering Biomaterials and Nanomedicine (CIBER-BBN) Campus de la UAB, 08193 Bellaterra, Spain

E-mail: $\underline{\text { mmas@icmab.es }}$

Dr. F. G. del Pozo

Universidad Técnica de Ambato, Av. Colombia y Chile, Ambato, Tubgurahua, Ecuador EC180105

Dr. T. Cramer, Prof. B. Fraboni

Department of Physics and Astronomy, University of Bologna, Viale Berti Pichat 6/2, 40127

Bologna, Italy.

+ These authors contributed equally to the work.

Keywords: Bar- Assisted Meniscus Shearing; Organic Field-Effect Transistors; Strain sensors; Scanning Kelvin Probe Microscopy; solution-processed organic semiconductors.

The influence of semiconductor morphology on the mechanical response of low voltage, flexible Organic Field-Effect Transistors fabricated on plastic substrates is explored. BarAssisted Meniscus Shearing is considered for patterning the organic semiconductor; morphological features of the film are changed by varying the shearing direction. Several devices with two different organic semiconductor coatings are reported. The response of such devices to two kinds of deformation, namely elongation and compression are investigated. The effect of mechanical stress is also considered. A detailed morphological analysis, including Atomic Force Microscopy and Scanning Kelvin Probe Microscopy, is performed in 
order to shine a light on the relationship between morphological features and electrical response of the devices. The results not only demonstrate that the response to bending is reliant on the morphological properties of the films, but also that it can be tuned according to the bar-shearing direction. The employment of the proposed approach for the development of devices with different extent of response to mechanical deformation, from bending sensors to strain-insensitive devices for applications needing electrical stability upon deformation, can be thus foreseen.

\section{Introduction}

Mechanical flexibility is surely one of the most demanded features in emerging electronic applications. Indeed, the possibility of conforming devices and entire electronic systems to non-planar surfaces could enable their actual integration on any kind of daily-life object. This would provide a significant step forward in the development of the Internet-of-Things (IoT) technologies [1] and finding applications in fields related to smart-packaging as well as for people and goods tracking.[2] Moreover, the mechanical robustness of electronic devices, i.e. the possibility to continuously or cyclically apply a deformation without significant variations in device performances, is a fundamental feature in applications ranging from the so-called “tattoo-electronics”[3]-[5] to wearable electronics and e-textiles.[6],[7]

Among the different technologies, organic electronics is generally considered the most suitable for the complete exploitation of the potentialities of flexible electronics. Organic materials can be solution-processed at low temperatures by means of cost-effective techniques,[8]-[9] thus being perfectly suitable for large-area fabrication of electronic devices on flexible substrates, including plastic,[10]-[12] paper [13] and fabric.[14]-[16]

Mechanical stability of organic electronic devices has been thoroughly explored in the last decade, in particular for Organic Field-Effect Transistors (OFETs), and it was found that, in this kind of structure, the mechanical sensitivity is mainly related to the morphological 
characteristics of the organic semiconductor film.[17]-[21] Indeed, by applying a mechanical deformation inducing an elongation of a layer from $L$ to $L+\Delta L$,a surface strain $\varepsilon=\Delta L / L$, is induced. In the case of thin-film devices, where the overall thickness of the device is negligible with respect to the one of the substrate $\left(t_{\text {sub }}\right)$, the thin-film strain $(\varepsilon)$ obtained by bending the substrate with a certain bending radius $R$ is given by the relationship

$$
\varepsilon=\frac{t_{\text {sub }}}{2 R}
$$

The thin-film strain determines a modification in the semiconductor morphology, thus affecting the charge carrier mobility inside the film.[16] Playing on the substrate thickness to maximise/minimise the bending sensitivity has been often proposed as a solution for fabricating bending sensors on one side, or mechanically-stable OFETs on the other side. Indeed, according to Equation 1, the thinner the substrate, the lower the thin-film strain is for a given bending radius. Nevertheless, the substrate thickness cannot be always arbitrarily chosen, but it is generally related to the employed fabrication equipment, and this is particularly true for those automated, large-area and high-throughput techniques, which must be mandatorily considered for the exploitation of flexible devices in real applications.

In this sense, the attention is being particularly focused on studying the way mechanical deformation affects the semiconductor morphology, and so what kind of morphological features should be obtained to modulate the bending response from high sensitivity to high stability, and in any case to ensure a significant mechanical robustness of devices. Amorphous semiconducting thin films are generally less sensitive than polycrystalline ones.[18] In the latter case, the influence of the processing parameters in determining the actual crystalline grain sizes has been considered.[20] However, this type of systematic study has hardly been realized on solution-processed organic semiconductor thin films, which are more appealing for industrial low-cost and large-area applications since they can be printed employing roll-toroll processes. Mechanical stability of solution-processed organic semiconductors has been 
explored in a number of publications,[22]-[25] but the impact of the thin-film morphology on the bending sensitivity, as well as the possibility of tuning this sensitivity by changing the morphological features of the deposited films, are substantially unexplored. As a possible cause, the generally poor control on the final morphological features of solution-processed organic semiconductors can be invoked.[26]

Among the large-area processing techniques compatible with roll-to-roll processes,[9] the Bar-Assisted Meniscus Shearing (BAMS) has attracted a significant interest thanks to its scalability and easiness-in-use.[27]-[29] Indeed, the importance of BAMS relays on its scalability, unlike other solution processing techniques such as drop-casting, spin-coating, or other methodologies that require long crystallization steps, combined with its simplicity, lowcost and in-air processing. Further, by tuning the deposition parameters, such as temperature, speed or solution, it is possible to modulate the thin-film morphology.[28] This method has proven to be useful to deposit uniform and reproducible crystalline semiconducting thin-films giving high-performance devices on inorganic Si/SiOx substrates [29] as well as on flexible plastic ones [30], in particular when the semiconductor was blended with insulating polymers. The strategy of employing blends combining a small molecule organic semiconductor with an insulating polymer has shown to enhance the material processability, the thin-film crystallinity and homogeneity, and the device environmental stability.[31]-[34]

In this paper, we report on the influence of mechanical deformation on low voltage OFETs fabricated on plastic substrates and employing BAMS for the deposition of the soluble organic semiconductor, namely 6,13-Bis(triisopropylsilylethynyl) pentacene (TIPS-PEN), blended with polystyrene (PS). In particular, the influence of the semiconductor thin-film morphology (tuned by changing the shearing direction) on the bending sensitivity was explored. Interestingly, we observed that each thin-film morphology exhibits an enhanced sensitivity to a specific deformation (i.e., elongation or compression) which was rationalized 
by Atomic Force Microscopy (AFM) and Scanning Kelvin Probe Microscopy (SKPM) characterization data.

\section{Results and Discussion}

\subsection{OFET fabrication and thin-film characterization}

As described in the Experimental Section, bottom-gate, bottom-contact OFETs based on a TIPS-PEN:PS blend were fabricated using PET flexible substrates and Parylene $\mathrm{C}$ as gate insulator (Figure 1). Blend solutions of TIPS-PEN and PS were prepared in a ratio 4:1 in chlorobenzene 2 wt\%. Then, the active layer was deposited by the BAMS solution- technique in ambient conditions, at a speed of $10 \mathrm{~mm} \mathrm{~s}^{-1}$ and keeping the substrates at $105{ }^{\circ} \mathrm{C}$ on the coating bed. The thin-film deposition was performed in two orthogonal directions, i.e., choosing the shearing direction to be either orthogonal or parallel to the channel length, as shown in Figure 1 (b) and (c), respectively. From now on, we will refer to these coatings as orthogonal (o-) or parallel (p-) coating. 


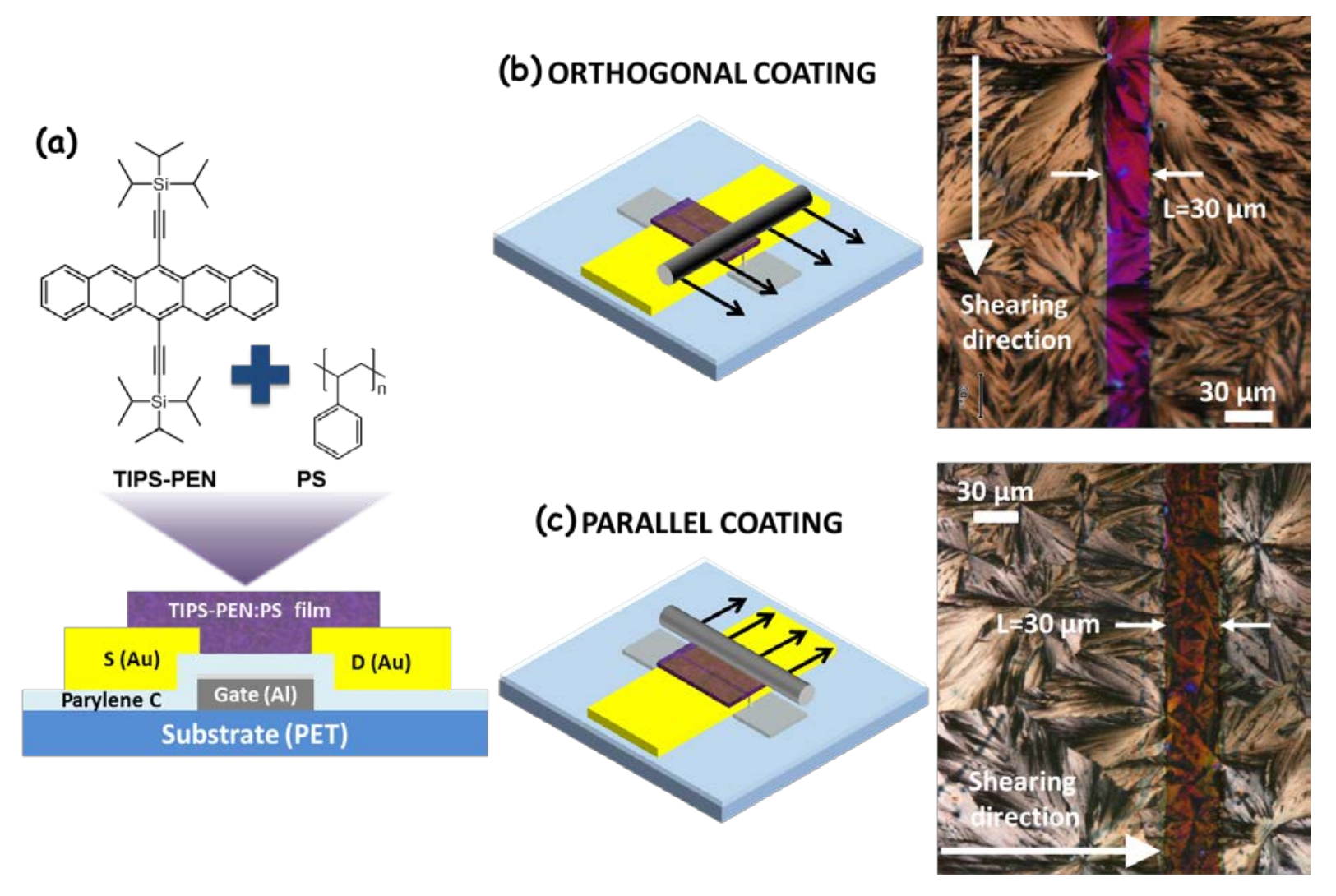

Figure 1. (a) Molecular structure of TIPS-PEN and PS, and a schematic representation of the Bottom-Gate Bottom-Contact OFET employed in this study. Representative sketches of the thin-film deposition by BAMS coating technique (left) and polarized optical microscopy images in the channel area for the orthogonal (b) and parallel (c) coatings.

From the polarized optical microscopy images of the prepared orthogonal and parallel coatings, shown in Figure 1 (b) and (c), respectively, it can be observed that highly crystalline and uniform films are formed on the substrate in both cases. However, for p-coated devices there is an evident difference in the size of the crystal domains between the channel area and the contact pads. As can be clearly observed from Figure 1 (c), the crystal domains dimensions are much smaller on the device channel than on the source and drain electrodes. On the other hand, for o-coated devices domains are very well connected from one pad to the other, through the channel. We believe that the reduction in domain size in the p-coating is related to the relatively high step between the gold contacts and the Parylene $\mathrm{C}$ (ranging 
around $60-80 \mathrm{~nm}$ ) and also to the different wettability of these materials, making the crystallization reliant on the surface. Interestingly, given the same device structure, materials and deposition parameters, a different crystallisation is obtained when the meniscus is moved parallel the channel width (o-coating) or parallel to the channel length (p-coating). Profilometry measurements allowed estimating the film thickness, which was found to be in the range of 100-200 nm (Figure S1). Both thin-film coatings were also characterized by Xray diffraction exhibiting identical diffraction patterns (Figure S2). Clear sets of (00l) reflections were observed, indicating the high degree of crystallinity of the deposited films and that crystallites are highly oriented with respect to the substrate. Also, the diffraction pattern agrees with the triclinic phase previously reported for TIPS-PEN.[35],[36]

\subsection{Electrical characteristics of BAMS-coated OFETs}

Before applying mechanical deformations on the fabricated devices, the pristine o-coated and p-coated OFETs were electrically characterized in air. Transfer and output characteristics were measured in a low voltage range, reaching $-5 \mathrm{~V}$ at maximum for both $V_{D S}$ and $V_{G S}$. Typical OFET transfer characteristics are shown in Figure 2 for both the orthogonal (a) and parallel (b) coating. As it can be noticed, a very small hysteresis was observed between the forward and reverse sweeps. Moreover, the output characteristics show no significant injection problems (see Figure S3). O-coated devices exhibited an average field-effect mobility of $\mu_{F E \text { sat }}=0.39 \pm 0.26 \mathrm{~cm}^{2} \mathrm{~V}^{-1} \mathrm{~s}^{-1}$, and a threshold voltage with an average value of $V_{t h}=-0.6 \pm 0.3 \mathrm{~V}$, both extracted in the saturation regime, and a mobility of $\mu_{F E \text {,lin }}=0.22 \pm 0.08 \mathrm{~cm}^{2} \mathrm{~V}^{-1} \mathrm{~s}^{-1}$ in the linear regime. Likewise, for $\mathrm{p}$-coated devices an average field-effect mobility of $\mu_{F E \text {,sat }}=0.32 \pm 0.17 \mathrm{~cm}^{2} \mathrm{~V}^{-1} \mathrm{~s}^{-1}$ and an average value of the threshold voltage of $V_{t h}=-1.1 \pm 0.4 \mathrm{~V}$ were extracted in saturation regime; the mobility in the linear regime was $\mu_{F E \text {,lin }}=0.13 \pm 0.05 \mathrm{~cm}^{2} \mathrm{~V}^{-1} \mathrm{~s}^{-1}$. It is noteworthy that for all devices the mobility has been extracted well above the subthreshold regime, in order to exclude mobility 
overestimation due to effects related to charge injection.[37] Both types of devices showed an on/off ratio on the order of $10^{4}-10^{5}$. These values have been calculated considering $15-20$ devices for each type of coating. It should be highlighted that the different thin-film morphology (i.e., crystalline domain size) between p- and o-coatings is not affecting the overall device performance, in terms of mobility values, which indicates that the crystallites are very well interconnected in both types of coating. However, p-coated devices showed a less steep switching, with a higher average subthreshold swing (SS) than o-coated devices (SS $=0.7 \pm 0.2 \mathrm{~V} \mathrm{dec}^{-1}$ and $S S=0.28 \pm 0.07 \mathrm{~V} \mathrm{dec}^{-1}$, for $\mathrm{p}$ - and o- coated devices, respectively). This difference in the subthreshold behavior can be related to the greater number of crystallites (and thus, grain boundaries) present in the p-coating.
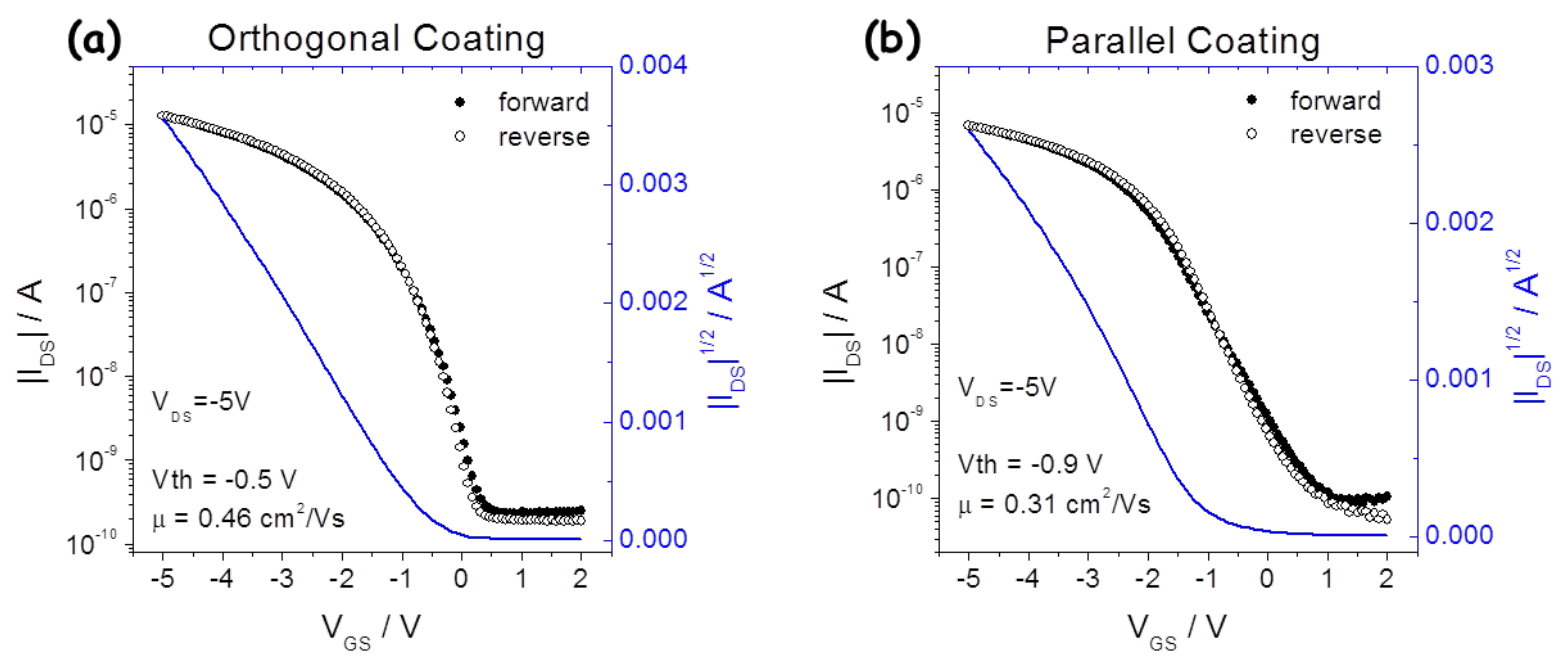

Figure 2. Transfer characteristics in the saturation regime and extraction of field effect mobility and threshold voltage of a typical o-coated (a) and p-coated (b) device. Closed and open symbols correspond to forward and reverse sweeps of $V_{G S}$, respectively.

\subsection{Response to strain and mechanical stability}

In order to investigate OFETs response to mechanical deformation, the variation of basic electronic parameters such as charge carrier mobility and threshold voltage upon bending was investigated. Two kinds of mechanical deformation were considered, i.e. elongation and 
compression. Elongation (compression) occurs when the substrate is convex (concave); this deformation determines an opposite dislocation of crystalline domains in the semiconductor films, thus determining respectively a decrease (in elongation) or an increase (in compression) of the charge carrier mobility. To characterize the extent of the deformation, the thin-film strain has been calculated (see Equation 1).

In the experiments, six different bending radii $(R=4.5 \mathrm{~cm}, 3 \mathrm{~cm}, 2 \mathrm{~cm}, 1 \mathrm{~cm}, 0.75 \mathrm{~cm}$ and $0.5 \mathrm{~cm}$ ) have been applied on the substrate, both in elongation and compression; six different values of thin-film strain $(|\varepsilon|=0.19 \%, 0.29 \%, 0.44 \%, 0.88 \%, 1.17 \%, 1.75 \%)$ have therefore been considered. Deformation was induced along the larger dimension of the device (substrate dimensions were $6 \times 2.5 \mathrm{~cm}$ ), parallel to the channel length, using laboratory clamps and reference cylinders of a reliable and reproducible determination of the bending radius. Being all the considered radii much larger than channel length, and substrate large enough to guarantee the application of a radial deformation on the channel area, the applied thin-film strain can be considered uniform. Results are reported on Figure 3 in terms of percentage mobility variation, $\Delta \mu / \mu_{0}=\left(\mu-\mu_{0}\right) / \mu_{0}$, being $\mu_{0}$ the value of charge carrier mobility of the pristine device while flat, and $\mu$ the value of mobility recorded while the device is bent. Each point is obtained as the average value of the mobility variation for at least three devices. As expected, when the device was elongated (Figure 3(a)), a mobility reduction was observed for both types of coating. The response of the devices is substantially linear, although o-coating resulted in a slightly larger sensitivity than p-coating. Interestingly enough, the difference between the two coatings is more evident when devices are compressed (Figure 3(b)). In this case, devices fabricated with orthogonal coating did not show a significant trend in their response: for a thin-film strain larger than $0.44 \%$, the mobility variation reached a plateau. On the contrary, devices with p-coated semiconductor showed a linear variation of the mobility over the whole range of applied strain, being significantly more sensitive to compression than 
devices with o-coated film. As regards threshold voltage, no substantial variations were observed for both coatings and both kinds of deformation: the absolute variation of threshold voltage is reported in Figure S4 in Supplementary Information. This result is not surprising,[19],[21] as mechanical deformation generally does not affect the semiconductor/insulator interface in a significant way.
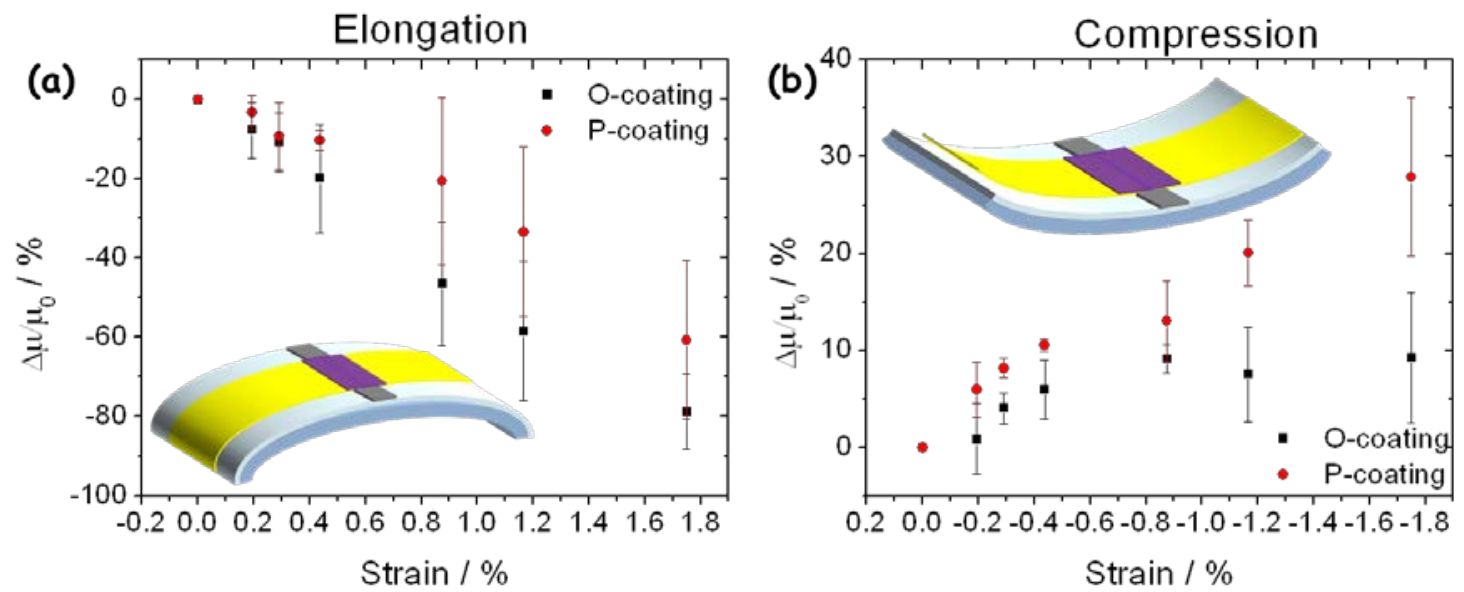

Figure 3. Average percentage mobility variation $\left(\Delta \mu / \mu_{0}\right)$ as a function of the applied strain $(|\varepsilon|$ $=0.19 \%, 0.29 \%, 0.44 \%, 0.88 \%, 1.17 \%, 1.75 \%)$ for devices with o-coated and p-coated organic semiconductor: (a) response to elongation; (b) response to compression. Average was calculated on a set of three devices at least.

As reported by previous literature,[38] the application of a deformation larger than a certain value can result in long standing or possibly permanent variations in the electrical properties of the device, related to permanent deformation of the substrate and/or to damages in the device structure. Ideally, when the device is brought back to a flat state after the application of a deformation, its electrical parameters should recover their original value. In order to characterize the critical value of strain for the different coatings, the devices have been brought back to flat state after the application of each deformation. Results are reported in Figure 4: the values of mobility variation are the same shown on Figure 3, and here referred to the original mobility value for the pristine, flat device. In the case of elongation (Figure 4(a)), a significant shift of the mobility value in flat condition towards lower values can be observed for both kinds of coating. In particular, after the application of a bending radius of 
$2 \mathrm{~cm}(|\varepsilon|=0.44 \%)$, error bars start to be significantly large, thus proving that devices do not recover from the applied stress in the same way. Despite the width of error bars, it is evident that the recovering ability of devices with o-coated semiconductor is averagely lower than the one of p-coated devices. Furthermore, after the application of a bending radius of $0.75 \mathrm{~cm}$ $(|\varepsilon|=1.17 \%)$, none of the investigated devices recovered the original mobility value. Interestingly enough, the effect of the stress is significantly lower when a compressive deformation is applied on the device (Figure 4(b)). In that case, and for both kinds of coating, the devices substantially recover the mobility value when brought back to flat condition after the application of the deformation. Also here, o-coated devices showed a larger variation in the flat condition than p-coated ones, but in this case the difference is less evident. According to the amplitude of error bars, a critical strain value $|\varepsilon|=1.17 \%(R=0.75 \mathrm{~cm})$ can be derived for both kinds of coating. Thus, films based on smaller crystallites (i.e., p-coating) seem to adapt more easily to deformations better recovering their pristine performance. Undoubtedly, the critical strain values found here could be correlated with the crystallinity of the TIPS-PEN active layer and with the plasticity of the PS layer.[39] However, this would require an indepth comparison between different structures and formulations, which is out of the scope of this work.
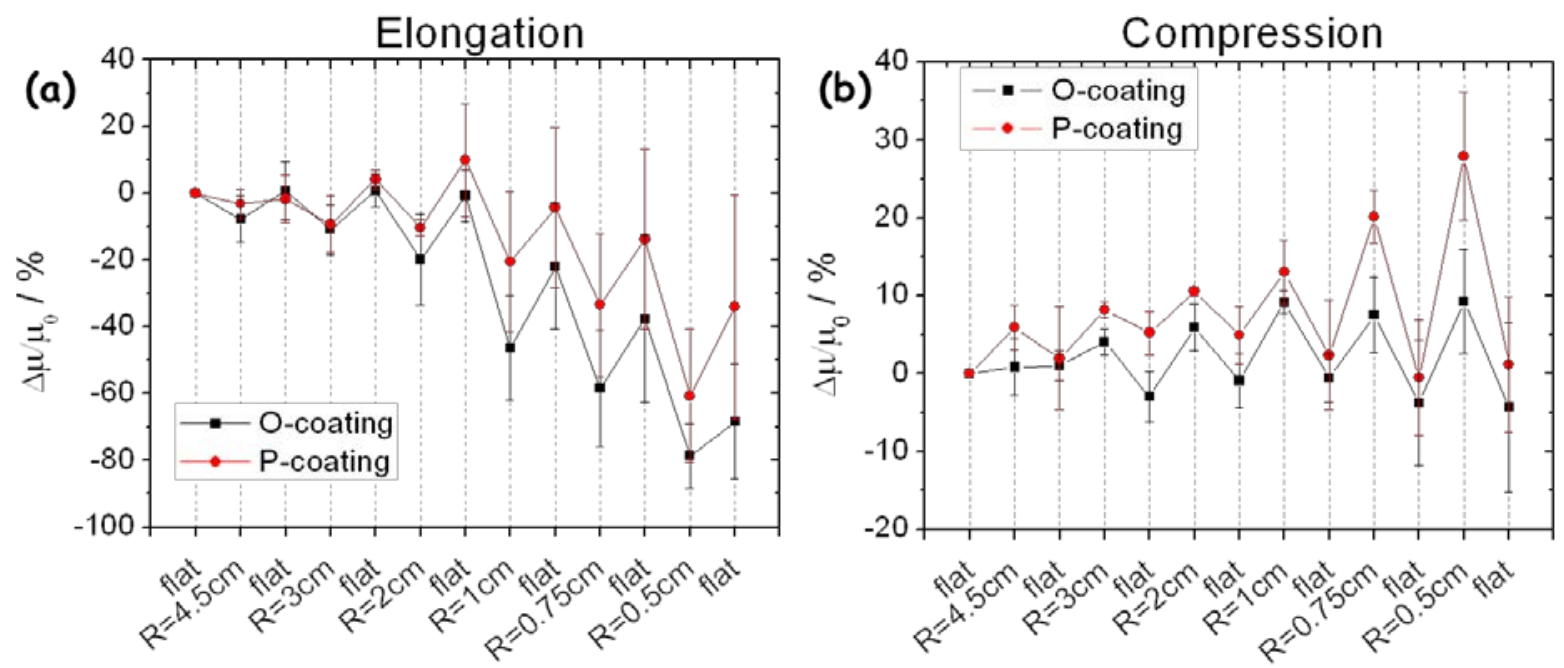

Figure 4. Average percentage mobility variation $\left(\Delta \mu / \mu_{0}\right)$ as a function of the applied bending radius $(R=4.5 \mathrm{~cm}, 3 \mathrm{~cm}, 2 \mathrm{~cm}, 1 \mathrm{~cm}, 0.75 \mathrm{~cm}, 0.5 \mathrm{~cm})$; after each deformation, devices were 
brought back to flat state. (a) response to elongation; (b) response to compression. The average was calculated on a set of at least three devices.

In order to further characterize the mechanical stress of devices with different kinds of coating, OFETs underwent several cycles of mechanical deformation applied with an automated system provided with a mechanical indenter. Each bending cycle consisted in the application of the mechanical deformation (elongation with $R=2 \mathrm{~cm}$, i.e. $\varepsilon=0.44 \%$ ) for 5 seconds and a relaxation time of 10 seconds. The transfer characteristic curves of the devices were recorded in the flat state for the pristine device, and after a certain number of deformations (100, 300, 600, 1000 cycles). Results are reported in Figure 5. For both kinds of coating, such a large number of cycles determined an evident shift of the characteristic curves. The threshold voltage is also affected by the mechanical strain, probably as a consequence of a modification of the insulator/semiconductor and/or electrode/semiconductor interface. In any case, such an effect is much more evident for devices with o-coated semiconductor: even after the first 100 cycles, both mobility and threshold voltage changed, while the following variations were much smaller. In particular, there is not a significant difference between the curves recorded after 600 and 1000 bending cycles. In the case of p-coated devices, more than 100 cycles were necessary to observe a significant shift of the curves, after that, the shift of the curves is substantially linear with the number of cycles. After 1000 bending cycles, a maximum shift of the threshold voltage of $700 \mathrm{mV}$ has been recorded for o-coated devices, while the shift was limited to $500 \mathrm{mV}$ in p-coated devices. 

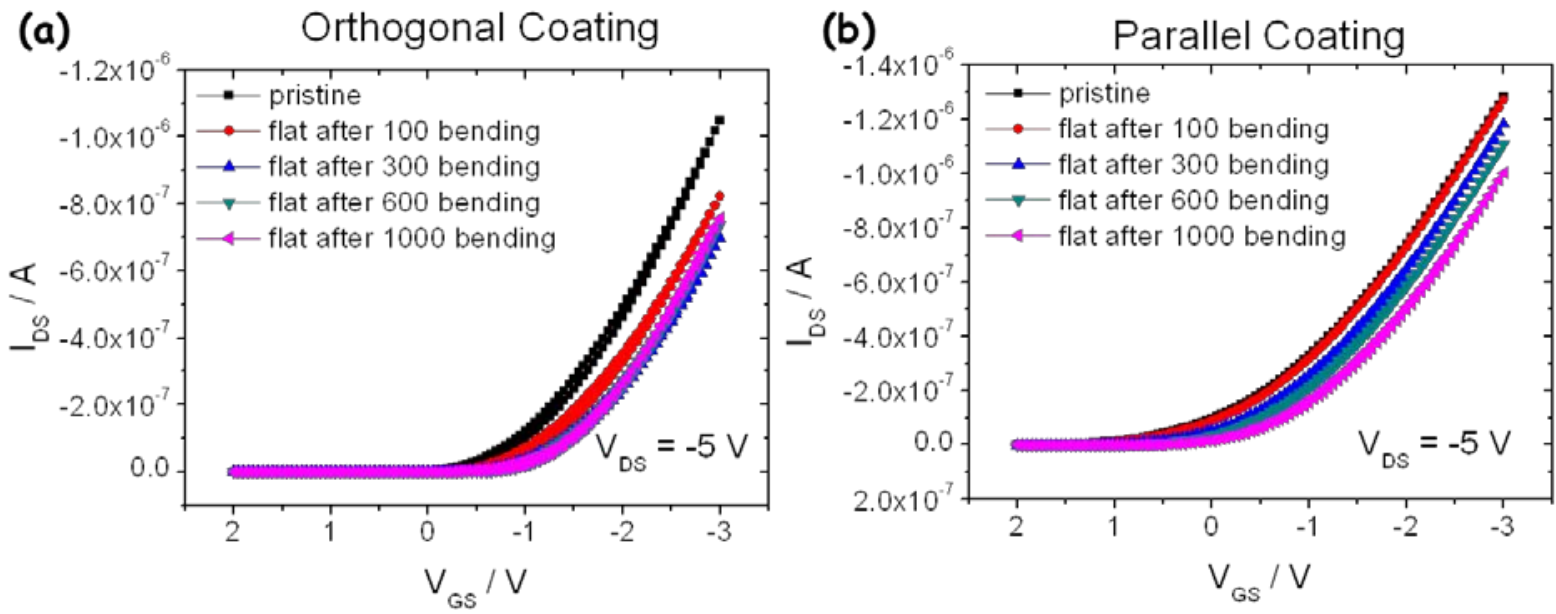

Figure 5. Transfer characteristic curves recorded for o-coated (a) and p-coated (b) devices after the application of an increasing number of elongations $(R=2 \mathrm{~cm}, \varepsilon=0.44 \%)$. The curves were recorded with the device in flat condition before the application of deformations (black squares), after 100 deformations (red dots), after 300 deformations (blue upward triangles), after 600 deformations (cyan downwards triangles) and after 1000 deformations (magenta leftward triangles).

\subsection{Morphological and local electrical characterization of strained devices}

In order to shine a light upon the relationship between the response to bending and mechanical stress and the morphology resulting from the different coating procedures, Atomic Force Microscopy (AFM) and Scanning Kelvin Probe Microscopy (SKPM) analysis have been carried out. SKPM gives access to the surface potential $V_{S P}$. In thin-film transistors with an exposed semiconducting channel, $V_{S P}$ is defined when the semiconductor becomes conductive, i.e. when doping or field effect induce charge carrier accumulation. In this case $V_{S P}$ follows the electrostatic potential in the accumulation layer plus an additional offset due to differences in work function with the material of the conductive AFM probe.[40],[41] During the SKPM scans, the transistors were kept at constant drain and gate bias of $V_{D S}=-1 \mathrm{~V}$ and $V_{G S}=-5 \mathrm{~V}$ to drive a current through the semiconducting channel. In this bias condition, the transistor operates in the linear regime and $V_{S P}$ follows the electrostatic potential in the accumulation layer in the semiconductor, thus providing information on local carrier concentration, variations in mobility and contact resistance.[40]-[42] 

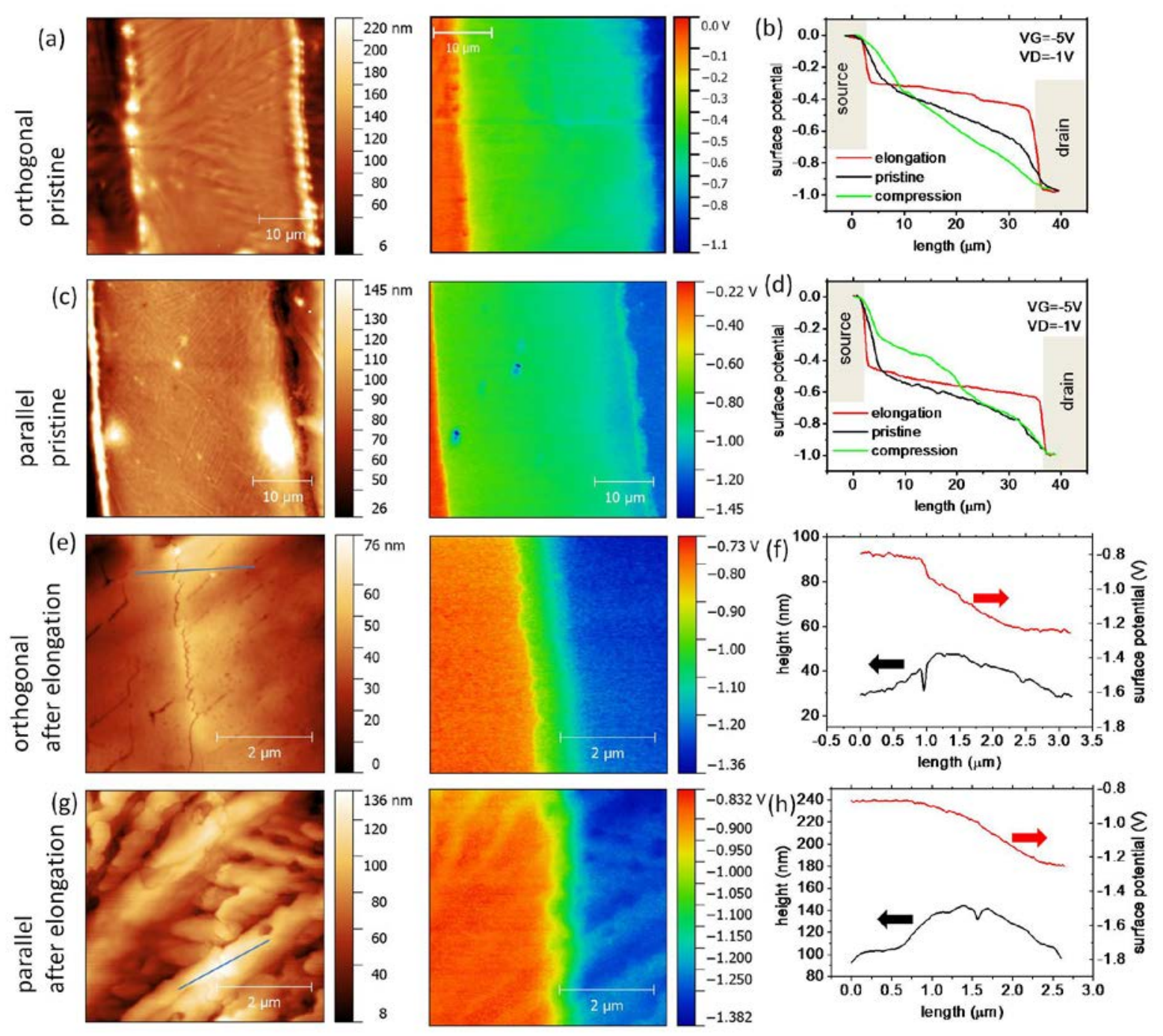

Figure 6. Investigation of microstructure and charge transport by Scanning Kelvin Probe Microscopy (SKPM). For each acquisition the topography image and the surface potential image are shown. (a,b) Images showing the whole channel obtained in pristine transistors with o-coating and corresponding profile of surface potential. For comparison surface potential profiles obtained after compression or elongation are added. (c,d) same but for p-coating. (e,f) SKPM images of the contact region in a o-coated transistor after elongation in higher resolution and graphs showing characteristic profiles. Images show that the presence of nanocracks causes the step in surface potential. (g,h) same but for p-coating. Here the cracks are less interconnected and the drop in surface potential is smaller.

Figure 6(a) and Figure 6(c) show the resulting maps of topography and $V_{S P}$ obtained on transistors fabricated with o- and p-coating. The topography of the o-coating exhibits features that are attributed to semiconducting microcrystals embedded in the PS matrix. In contrast, 
the p-coating contains smaller surface structures hinting to an overall smaller feature size of microcrystals embedded in the polymer matrix. Images show that, for both types of coatings, the semiconductor layer is thicker in the channel area than on the electrodes. Moreover, an accumulation of material is observed at the electrodes' contact, thus leading to a significant increase in surface height at the electrodes/channel interface. This accumulation of material in the spatially confined channel is attributed to the larger affinity of the TIPS-PEN:PS ink to the Parylene surface in the channel instead of the gold surface exposed on electrodes. Overall, both coatings show a very homogeneous surface topography inside the channel, resulting from the successful embedding of semiconducting micro-crystals in the polymer matrix.

In the $V_{S P}$ maps, the electrodes can be identified as isopotential surfaces at $0 \mathrm{~V}$ and $-1 \mathrm{~V}$. For both kinds of coatings, the potential drops significantly at the channel/electrode interface due to contact resistance effects, whereas a homogeneous decrease in potential is observed along the channel. Local inhomogeneties indicating high electric fields due to traps or transport barriers are not present. The potential profiles along the channel from these $V_{S P}$-maps of pristine transistors are shown in Figure 6(b) and Figure 6(d). In addition, we show profiles obtained from $V_{S P}$ maps of transistors that were subjected to compression (with a maximum strain value $\varepsilon=-1.75 \%$ ) or elongation (with a maximum strain value $\varepsilon=1.75 \%$ ) and then repositioned in the flat geometry for the SKPM characterization. Two important observations can be made from these graphs: (i) in the elongated transistors a strong increase in contact resistance is observed. Almost all the potential variation occurs at the semiconductor/electrode interfaces and shows that a strong electric field is required here to inject or extract carriers from the semiconductor. (ii) Compression has the opposite effect: the contact resistances are reduced. However, stronger variations in slope of the $V_{S P}$ profile are observed in the channel, indicating the formation of local barriers for charge carrier transport. It is possible to observe that the contact resistance for a pristine device is larger in p-coated devices than in o-coated ones, probably as a consequence of the morphology variation at the 
electrode/channel interface. Moreover, o-coated devices showed an increase of contact resistance larger than p-coated ones during elongation, justifying the larger current reduction. In contrast, p-coated devices showed a larger contact resistance reduction than o-coated ones as a response of compression, thus explaining the larger sensitivity of p-coated devices to this kind of deformation. Detailed SKPM images of the electrode contact region, as shown in Figure 6(e) and Figure 6(g) obtained after elongation of o- and p-coated transistors, reveal the reason for the contact resistance increase. The smooth surface morphology of the o-coating is interrupted by nano-cracks that extend in the direction orthogonal to the current. Coincident with these cracks, a strong decrease in surface potential is observed (Figure 6(f)). A similar finding is made on the parallel coating (Figure 6(h)). However, in this case the coating is less homogeneous and smaller microcrystals can be identified. Close inspection of the image reveals again the presence of small cracks that, however, do not extent linearly in a single direction but are more distributed and not fully interconnected. Again, at the location of the cracks, a decrease in surface potential is observed, although smaller in magnitude.

We attribute the crack formation at the electrode contacts to local strain maxima that occur due to the presence of a surface step probably caused by the thickness of bottom contact source and drain electrodes. As microcrystals have to adapt to this step in surface height at the interface of electrodes, a pre-strain is already present in the pristine, flat state. Elongation leads to an increase of strain and when a critical value is reached, strain localization and crack opening occurs. Such a crack formation is irreversible, and cracks do not close when the transistor is returned into the flat state. The smaller crystallites found in the parallel coating lead to a distributed crack opening and cracks are less interconnected. As a consequence, some transport pathways remain open and degradation due to strain is less severe. This interpretation can also explain the different dynamic of electrical characteristic degradation observed in the mechanical stress test (cfr. Figure 5 and related text). Indeed, the large shift observed in o-coated devices after the first 100 bending cycles can be explained with the 
formation of the majority of the deep cracks in this stage of the experiment, due to the large dimensions of crystals which result is a certain stiffness of the active layer. In p-coated devices, the smaller dimensions of crystals contribute to enhance elasticity of the active layer: the cracks are thus not only scarcely connected, but are formed over a more prolonged stress and also uniformly with the number of deformations.

\section{Conclusion}

We explored the employment of Bar-Assisted Meniscus Shearing as technique for the deposition of the organic semiconductor in order to tailor the response of low voltage organic field-effect transistors to mechanical deformation. By changing the shearing direction, two different thin-film morphologies were obtained: these coatings, namely orthogonal and parallel coating, resulted in similar electrical characteristics, but more importantly they showed different electrical responses to different kinds of mechanical stress, namely elongation and compression. In particular, it was demonstrated that elongation generally resulted in a larger stress for the devices than compression, especially for those based on films with larger crystallites (i.e., orthogonal coating). In contrast, for compression films with smaller crystals (i.e., parallel coating) showed a larger sensitivity than those fabricated by orthogonal coating. Morphological and SKPM characterization provided a clear explanation for the electrical response to deformation: an increase (a decrease) of the contact resistance was observed for elongated (compressed) devices, thus justifying the observed current decrease (increase). The extent of such contact resistance variations justifies the different performances obtained for o-coated and p-coated devices in elongation and compression. Moreover, for both kinds of coating, the formation of cracks in the semiconductor film was observed, but these cracks were more interconnected in the case of orthogonal coating, thus justifying the larger effects of mechanical stress observed in this case. The results here reported represent a first step for the investigation of BAMS as technique for fabricating 
flexible OFETs whose sensitivity to mechanical deformation may be modulated; other parameters possibly influencing this property, such as shearing speed and ink composition, will be investigated in further research.

\section{Experimental Section}

Device Fabrication: Devices were fabricated onto $175 \mu$ m-thick polyethylene terephthalate (PET, Goodfellow) substrates $(6 \times 2.5 \mathrm{~cm})$, which were cleaned with multiple rinsing of acetone, isopropanol and deionized waters and dried with nitrogen. An aluminum layer (nominal thickness of 100nm) was then deposited by means of thermal evaporation (Pressure: $5 \times 10^{-5}$ Torr). The shape of the gate was then defined by means of photolithography. A nano-sized aluminum oxide layer (nominal thickness of $8 \mathrm{~nm}$ ) was grown on the gate by an annealing process, storing the substrate overnight at a temperature of $50^{\circ} \mathrm{C}$. After that, a

Parylene C (Specialty Coating Systems) layer was deposited by means of Chemical Vapor Deposition (CVD); the nominal thickness of this layer is $170 \mathrm{~nm}$. Afterwards, a photoresist was patterned onto the gate electrode using a self-alignment process. A gold layer with a nominal thickness of $60 \mathrm{~nm}$ was thermally evaporated onto the substrate under a pressure of $5 \times 10^{-5}$ Torr using a shadow mask. The excess of gold, i.e. the part deposited onto the photoresist layer covering the channel, was removed by lift-off, thus defining source and drain in the channel area and determining a minimum overlap between source and drain and the gate electrode. The channel width and length of the fabricated electrodes was $30 \mu \mathrm{m}$ and 5 mm respectively.

TIPS-PEN and PS of molecular weight $M_{w}=10000 \mathrm{~g} / \mathrm{mol}$ were purchased from Ossila and Sigma-Aldrich, respectively, and used without further purification. Blend solutions were prepared in anhydrous chlorobenzene (2\% wt.) at a weight ratio of 4:1 (TIPS-PEN:PS). Before the thin-film deposition, substrates were gently cleaned by high performance liquid chromatography (HPLC) quality acetone and isopropanol and then dried under nitrogen. The 
gold surface of the source and drain electrodes was chemically modified with a selfassembled monolayer (SAM) of pentafluorobenzenethiol (PFBT, Sigma-Aldrich), by immersing the substrates in a $15 \mathrm{mM}$ solution of PFBT in isopropanol for 30 minutes. Finally, the substrates were rinsed with more pure isopropanol and dried under nitrogen. TIPS-PEN:PS thin-films were deposited at ambient conditions using a home-designed bar coater with a cylindrical Teflon bar. The substrates were kept at $105^{\circ} \mathrm{C}$ on the coating bed, as well as the blend solution. About $15 \mu \mathrm{l}$ of the blend solution were dispensed between the substrate and the bar, and immediately sheared at speed of $10 \mathrm{~mm} \mathrm{~s}^{-1}$.

Thin-Film Characterization: Optical microscope pictures were taken using an Olympus BX51 equipped with polarizer and analyzer. X-ray diffraction measurements were carried out with a PANalytical X'PERT MRD system. The profile of the thin-films was characterized using a Dektak XT ${ }^{\mathrm{TM}}$ stylus profiler (Bruker).

Electrical Characterization: Electrical measurements were performed using an Agilent B1500A semiconductor device analyzer connected to the samples with a Karl SÜSS probe station, at ambient conditions. The field-effect mobility in the saturation regime was extracted using the relationship

$$
\mu_{F E, s a t}=\frac{2 L}{W C} \times\left(\frac{\partial \sqrt{\left|I_{S D, s a t}\right|}}{\partial V_{S G}}\right)^{2}
$$

where $C$ is the insulator capacitance per unit area, and $W$ and $L$ are the width and length of the channel, respectively. Field-effect mobility in the in the linear regime was extracted as

$$
\mu_{F E, \text { lin }}=\frac{L}{W C V_{D S}} \times\left(\frac{\partial\left|I_{S D, \text { lin }}\right|}{\partial V_{S G}}\right)
$$

Bending Characterization: During bending tests, transfer characteristic curves have been acquired using a Keithley 2636 SourceMeter ${ }^{\circledR}$, controlled by a custom-made Matlab® software. Stress tests were carried out using a system for the application of controlled force 
(Imada Digital Force Measurement Gauge); a mechanical indenter was employed to apply the deformation to the substrate.

Scanning Kelvin Probe Microscopy: Images were acquired with a Park NX10 setup using PPP-NCST-Au probes (Nanosensors). Height and surface potential images were acquired in parallel using a setup with two lockin amplifiers. The first amplifier was locked on a resonance frequency of the cantilever $(718 \mathrm{kHz})$ and the second at the frequency of the AC signal applied to the tip by a function generator $(1 \mathrm{~V}, 17 \mathrm{kHz})$. The DC potential applied to the tip by the SKPM feedback to nullify the electrostatic modulation was sampled as the surface potential map. During measurements bias was applied to transistor source, drain and gate contacts using a Keysight 2912B source measure unit.

\section{Acknowledgements}

This work was funded by the ERC StG 2012-306826 e-GAMES, Networking Research Center on Bioengineering, Biomaterials, and Nanomedicine (CIBER-BBN), the DGI (Spain) project FANCY CTQ2016-80030-R, the Generalitat de Catalunya (2014-SGR-17) and the Spanish Ministry of Economy and Competitiveness, through the "Severo Ochoa" Programme for Centers of Excellence in R\&D (SEV-2015-0496). I. T. acknowledges FPU fellowship from the Ministry and is enrolled in the Materials Science PhD Program of Universitat Autònoma de Barcelona. F. G. Del Pozo thanks Universidad Técnica de Ambato and Secretaría de Educación Superior, Ciencia, Tecnología e Innovación for funding through a doctoral scholarship “Convocatoria abierta 2010.”

\section{References}

[1] Y. Zhan, Y. Mei, L. Zheng, J. Mater. Chem. C 2014, 2.7, 220.

[2] L. M. Dumitru, M. Irimia-Vladu, and N. S. Sariciftci, Comprehensive Analytical Chemistry 2016, 74, 247.

[3] F. Greco, A. Zucca, S. Taccola, B. Mazzolai, V. Mattoli, ACS Appl. Mater. Interfaces 2013, 59461.

[4] A. Reuveny, S. Lee, T. Yokota, H. Fuketa, C. M. Siket, S. Lee, T. Sekitani, T. Sakurai, S. Bauer, T. Someya, Adv. Mater. 2016, 28, 3298. 
[5] R. A. Nawrocki, N. Matsuhisa, T. Yokota, T. Someya, Adv. Electron. Mater. 2016, 2, 1500452.

[6] X. Wang, Z. Liu, T. Zhang., Small 2017, 1602790.

[7] Y. Q. Li, P. Huang, W. B. Zhu, S. Y. Fu, N. Hu, K. Liao, Sci. Rep, 2017, 7, 45013.

[8] D. R. Gamota, P. Brazis, K. Kalyanasundaram, J. Zhang, Printed organic and molecular electronics, Springer Science \& Business Media, New York, NY, USA 2013.

[9] Y. Xu, C. Liu, D. Khim, Y. Y. Noh, Phys. Chem. Chem. Phys. 2015, 17, 26553.

[10] S. Conti, S. Lai, P. Cosseddu, A. Bonfiglio, Adv. Mater. Technol. 2017, 2, 1600212 .

[11] G. Grau, V. Subramanian, Adv. Electron. Mater. 2016, 2, 1500328.

[12] L. Feng, C. Jiang, H. Ma, X. Guo, A. Nathan, Org. Electron. 2016, 38, 186.

[13] T. Liang, X. Zou, A. D. Mazzeo Proc. SPIE 2016, 9836.

[14] M. Maccioni, E. Orgiu, P. Cosseddu, S. Locci, A. Bonfiglio, 3rd IEEE/EMBS International Summer School on Medical Devices and Biosensors, 2006, 5.

[15] G. Mattana, P. Cosseddu, B. Fraboni, G. G. Malliaras, J. P. Hinestroza, A. Bonfiglio, Org. Electron. 2011, 12, 2033.

[16] W. Kim, S. Kwon, S. -M. Lee, J. Y. Kim, Y. Han, E. Kim, K. C. Choi, S. Park, B. -C. Park, Org. Electron. 2013, 14, 3007.

[17] H. Gleskova, S- Wagner, W. Soboyejo, Z. Suo, J. Appl. Phys. 2002, 92, 6224.

[18] T. Someya, T. Sekitani, S. Iba, Y. Kato, H. Kawaguchi, T. Sakurai, Proc. Natl. Acad. Sci. USA 2004, 101, 9966. 
[19] P. Cosseddu, S. Milita, A. Bonfiglio, IEEE Electron Device Lett. 2012,33, 113.

[20] V. Scenev, P. Cosseddu, A. Bonfiglio, I. Salzmann, N. Severin, M. Oehzelt, N. Koch, J.P. Rabe, Org. Electron. 2013, 14, 1323.

[21] P. Cosseddu, G. Tiddia, S. Milita, A. Bonfiglio, Org. Electron. 2013, 14, 206.

[22] D. K. Hwang, C. Fuentes-Hernandez, J. B. Kim, W. J. Potscavage, B. Kippelen, Org. Electron. 2011, 12, 1108.

[23] V. Raghuwanshi, D. Bharti, S. P. Tiwari, Org. Electron. 2016, 31, 177.

[24] V. Raghuwanshi, D. Bharti, I. Varun, A. K. Mahato, S. P. Tiwari, Org. Electron. 2016, 34, 284.

[25] T. Sekine, D. Kumaki, S. Tokito, Materials 2017, 10, 18.

[26] M. Mas-Torrent, C. Rovira, Chem. Rev. 2011, 111, 4833.

[27] I. Temiño, F. G. del Pozo, M. R. Ajayakumar, S. Galindo, J. Puigdollers, M. Mas-Torrent, Adv. Mater. Technol. 2016, 1600090.

[28] S. Galindo, A. Tamayo, F. Leonardi, M. Mas-Torrent, Adv. Funct. Mater. 2017, doi: 10.1002/adfm.201700526.

[29] F. G. del Pozo, S. Fabiano, R. Pfattner, S. Georgakopoulos, S. Galindo, X. Liu, S. Braun, M. Fahlman, J. Veciana, C. Rovira, X. Crispin, M. Berggren, M. MasTorrent, Adv. Funct. Mater. 2016, 26, 2379.

[30] S. Georgakopoulos, F. G. del Pozo, M. Mas-Torrent, J. Mater. Chem. C 2015, 3,12199. 
[31] M. R. Niazi, R. Li, E. Qiang Li, A. R. Kirmani, M. Abdelsamie, Q. Wang, W. Pan, M. M. Payne, J. E. Anthony, D.-M. Smilgies, S. T. Thoroddsen, E. P. Giannelis, A. Amassian, Nat. Commun. 2015, 6, 8598.

[32] R. Hamilton, J. Smith, S. Ogier, M. Heeney, J. E. Anthony, I. Mcculloch, J. Veres, D. D. C. Bradley, T. D. Anthopoulos, Adv. Mater. 2009, 21, 1166.

[33] Lee, W.H.; Park, Y.D, Polymers 2014, 6, 1057.

[34] Q. Zhang, F. Leonardi, S. Casalini, I. Temiño, M. Mas-Torrent, Sci. Rep. 2016, 6, 39623.

[35] J. E. Anthony, J. S. Brooks, D. L. Eaton, S. R. Parkin, J. Am. Chem. Soc. 2001 , $123,9482$.

[36] S. C. B. Mannsfeld, M. L. Tang, Z. Bao, Adv. Mater. 2011, 23, 127.

[37] I. McCulloch, A. Salleo, M. Chabinyc, Science 2016, 352, 1521.

[38] T. Cramer, L. Travaglini, S. Lai, L. Patruno, S. de Miranda, A. Bonfiglio, P. Cosseddu, B. Fraboni, Sci. Rep. 2016, 6, 38203.

[39] B. J. Gurmessa, A. B. Croll, Phys. Rev. Lett. 2013, 110, 074301.

[40] L. Bürgi, H. Sirringhaus, R. H. Friend, Appl. Phys. Lett. 2002, 80, 2913.

[41] S. Almubayedh, M.Sc. Degree Dissertation, Laurentian University of Sudbury (Ontario, Canada), Sept. 2014.

[42] V. Palermo, M. Palma, P. Samorì, Adv. Mater. 2006, 18, 145. 
